\title{
Analysis of 809 Facial Bone Fractures in a Pediatric and Adolescent Population
}

\author{
Sang Hun Kim, Soo Hyang Lee, Pil Dong Cho \\ Department of Plastic and Reconstructive Surgery, Ilsan Paik Hospital, Inje University College of Medicine, Goyang, Korea
}

Background Facial fractures are infrequent in children and adolescents and have different clinical features from those in adults. The low incidence in children and adolescents reflects the flexibility and underdevelopment of their facial skeletons, as well as their more protected environments. Only a few reports have reviewed such patients in Korea. The authors performed a retrospective study to analyze the characteristics of facial fractures in the Korean pediatric population.

Methods We conducted a retrospective review on a series of 741 patients, aged $<18$ years, with facial fractures who had been treated at our hospital between 2006 and 2010. The following parameters were evaluated: age, sex, cause, location and type of fractures, associated injuries, treatment and complications.

Results A total of 741 consecutive patients met the inclusion criteria. The ratio of boys to girls was 5.7:1. Facial fractures most commonly occurred in patients between 13 and 15 years of age (36.3\%). The most common causes of injury was violence. The nasal fracture was the most common type of fracture (69\%) and the blowout fracture was the second most common (20\%). Associated injuries occurred in 156 patients (21\%).

Conclusions The incidence of pediatric facial fractures caused by violence is high in Korea. Our results show that as age increases, etiological factors and fracture patterns gradually shift towards those found in adults. This study provides an overview of facial fractures in these age groups that helps illustrate the trends and characteristics of the fractures and may be helpful in further evaluation and management.

Keywords Facial bones / Adolescent / Violence

\author{
Correspondence: Pil Dong Cho \\ Department of Plastic and \\ Reconstructive Surgery, Ilsan Paik \\ Hospital, Inje University College of \\ Medicine, 170 Juhwa-ro, Ilsanseo-gu, \\ Goyang 411-706, Korea \\ Tel: +82-31-910-7320 \\ Fax: +82-31-910-7814 \\ E-mail: pildong@naver.com
}

No potential conflict of interest relevant to this article was reported.

\section{INTRODUCTION}

As compared to adults, fractures of the facial skeleton are rare in the pediatric age group. Retrospective studies of maxillofacial trauma, involving both adults and children, have demonstrated that less than $15 \%$ of such fractures occurs in those younger than 16 years of age and less than $1 \%$ of all facial fractures occurs in children younger than 5 years of age $[1,2]$.
Pediatric facial bones are protected by children's low face-tohead volume ratio, which allows the cranium, rather than the face, to absorb most of the traumatic impact. Furthermore, children's facial bones are more resistant to fractures due to their higher elasticity, lack of sinus pneumatization, thicker bony walls surrounded by adipose tissue, and stabilization of the mandible and maxilla by unerupted teeth [3]. Young children are usually in protected environments under parental supervision and are consequently less 
likely to be exposed to the major injuries, occupational trauma, or interpersonal violence that are typical of adult facial fractures $[4,5]$. Understanding of the causes, severity, and specific characteristics of pediatric fractures is essential for preventing esthetic and functional deficits and will improve our ability to prevent such injuries [6].

The purpose of the present study was to provide a comprehensive overview of facial trauma in the Korean pediatric and adolescent population and to assist surgeons in further assessment and proper management.

\section{METHODS}

We retrospectively analyzed the medical records of 809 facial fractures in 741 patients aged 18 years or younger who had been treated at our hospital between 2006 and 2010.

Each patient sustained a fracture of one or more facial bones, which included the nasal bone, maxilla, orbit, zygoma, and mandible. Fractures were confirmed by computed tomography (CT). Patients were divided into 6 age groups: the 0 - to 3-year-old, 4 - to 6-year-old, 7- to 9-year-old, 10- to 12-year-old, 13- to 15-year-old, and 16- to 18-year-old groups. The following analyses were conducted for each age group: causes of injury (e.g., motor vehicle accidents, falling, bicycle accidents, violence, and sports), fracture type (e.g., nasal, orbital, zygomaticomaxillary, mandibular, and Le Fort fractures), associated injuries, and clinical outcomes.

Fig. 1. Annual distribution of the facial fractures of the 741 patients

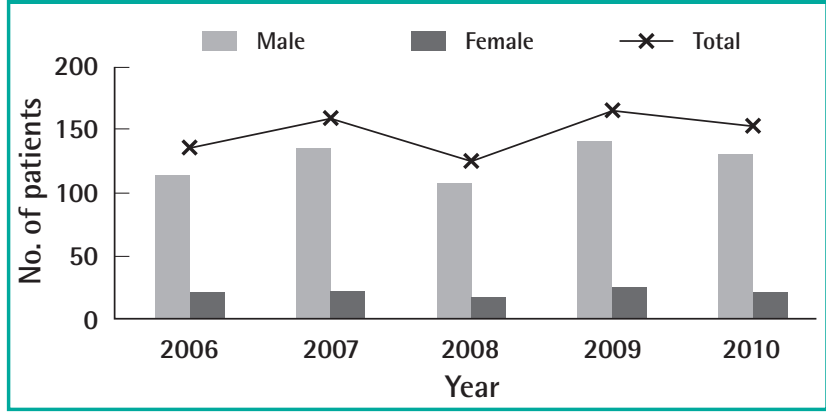

\section{Fig. 2. Distribution of patients by sex in each age group}

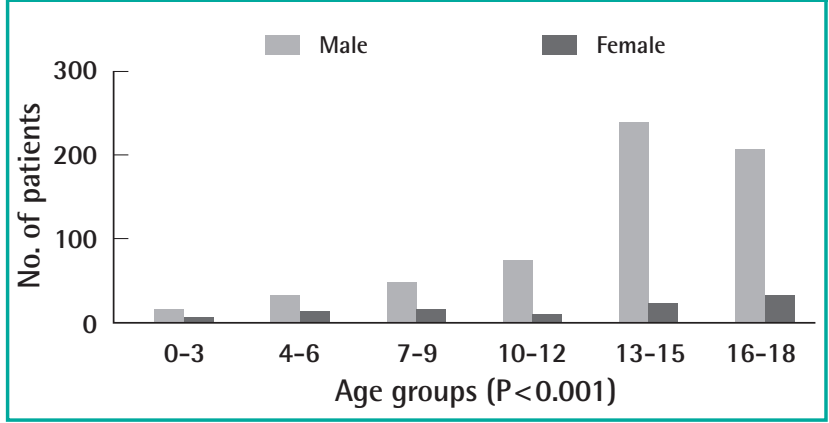

Patient characteristics were analyzed using descriptive statistics and comparisons were made with the chi-squared test. A P-value of less than 0.05 was considered statistically significant.

\section{RESULTS}

\section{Patient characteristics}

A total of 741 patients were evaluated for the study. The mean age was 13.0 years (standard deviation [SD], 4.1 years). The annual distribution of patients during the 5-year period is shown in Fig. 1. There was no significant difference in the number of patients among the individual years. The overall ratio of males to females was 5.7:1. The ratio of males to females varied considerably among the age groups (Fig. 2). Older children were significantly more likely to sustain facial fractures $(\mathrm{P}<0.001)$. Facial fractures occurred most frequently in the 13 to 15 age group (36.3\%). The monthly distribution of facial fractures is shown in Fig. 3. The number of patients with facial fractures was the lowest during January $(n=29)$, increased in the spring and fall months, and peaked in October $(n=88)$. More than half (50.8\%) of the patients visited our hospital between $1 \mathrm{PM}$ and 6 PM (Fig. 4).

\section{Cause of injury}

The most common cause of injury was violence ( $n=278,38 \%)$, followed by falls $(n=227,31 \%)$, and sports $(n=123,17 \%)$ (Table 1). The mean age of the patients involved in violence was 14.4 years (SD, 2.5 years), while the mean age of those who

\section{Fig. 3. Monthly distribution of facial injuries}

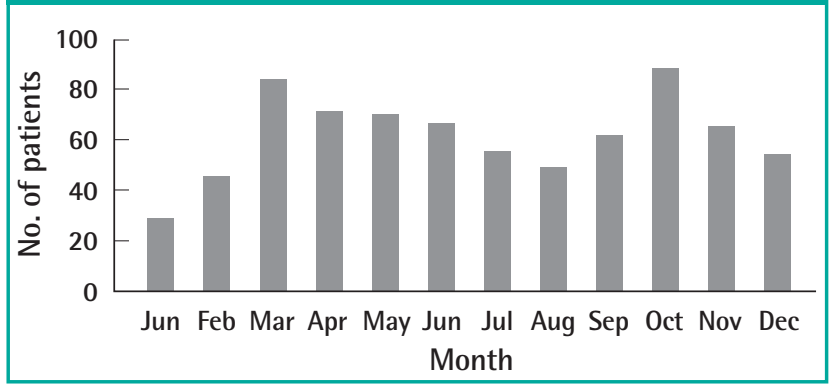

Fig. 4. Hours of emergency care

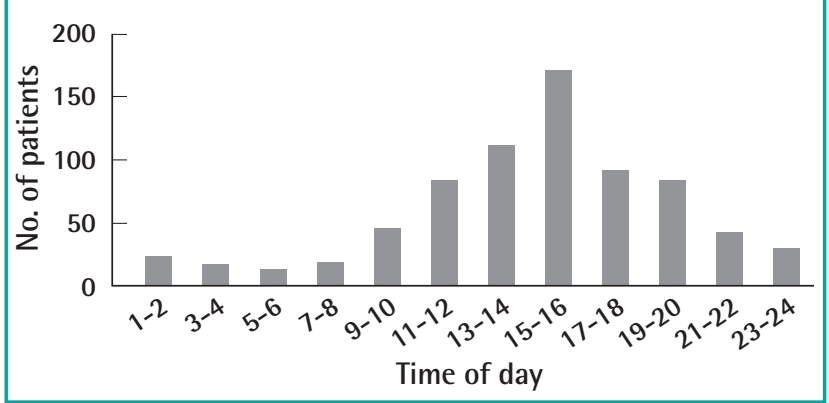


Table 1. Causes of injury and distribution in each age group

\begin{tabular}{|c|c|c|c|c|c|c|c|}
\hline \multirow{2}{*}{ Type of accident } & \multirow{2}{*}{$\begin{array}{l}\text { Number of } \\
\text { patients }(\%)\end{array}$} & \multicolumn{6}{|c|}{ Age group (yr) } \\
\hline & & $0-3$ & $4-6$ & $7-9$ & $10-12$ & $13-15$ & $16-18$ \\
\hline Violence & 278 (38) & 1 & 2 & 9 & 33 & 134 & 99 \\
\hline Falling & 227 (31) & 25 & 34 & 26 & 38 & 53 & 52 \\
\hline Sports & $123(17)$ & - & 1 & 4 & 17 & 52 & 49 \\
\hline Motor vehicle & $60(8)$ & - & 7 & 7 & 7 & 12 & 27 \\
\hline Car & $22(3)$ & - & 6 & 1 & 4 & 3 & 7 \\
\hline Motorbike & $15(2)$ & - & - & - & - & 2 & 13 \\
\hline Car-pedestrian & $24(3)$ & - & 1 & 6 & 3 & 7 & 7 \\
\hline Bicycle & $31(4)$ & & 2 & 9 & 3 & 10 & 7 \\
\hline Others & $21(3)$ & 1 & 1 & 2 & 3 & 7 & 7 \\
\hline Total & $741(100)$ & 27 & 47 & 69 & 88 & 269 & 241 \\
\hline
\end{tabular}

Fig. 5. Locations of the 809 facial fractures in the 741 patients

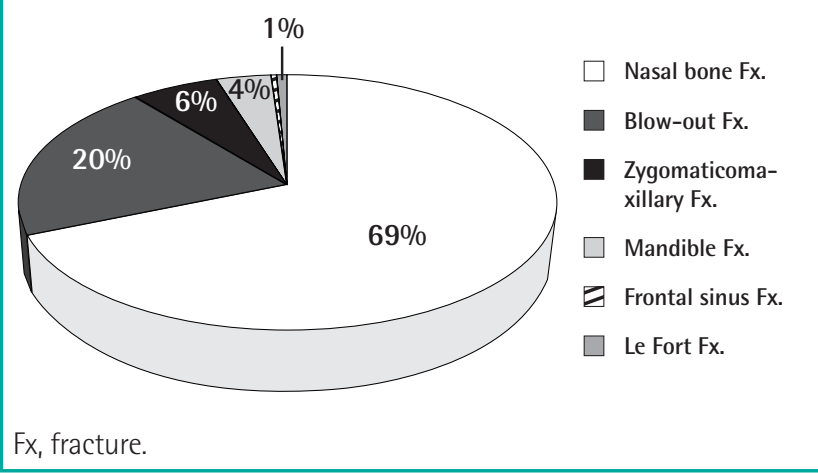

Fig. 6. Distribution of facial fracture types in each age group patients

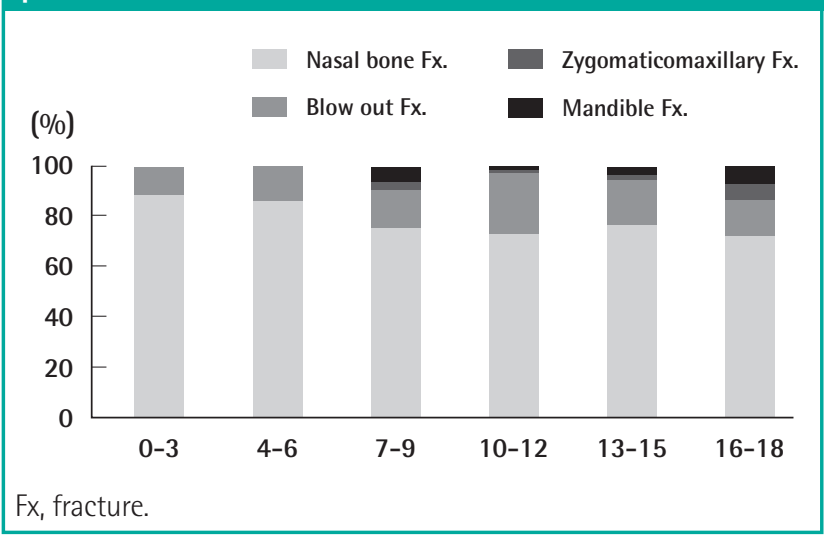

were not involved in violence was 12.2 years (SD, 4.7 years). Approximately $91 \%$ of the patients involved in violence were male. Falling was a common cause of injury in children younger than 12 years of age. Violence was more common in those older than 13 years of age.

\section{Localization and type of injuries}

Nasal bone fractures were the most common among all facial bone fractures, accounting for $69 \%$ of all fractures, and the incidence decreased with age (Figs. 5, 6). Orbital fractures (20\%) and zygomaticomaxillary fractures $(6 \%)$ were the next most common. The medial wall was the most frequently fractured site among orbital fractures, accounting for about $48 \%$ of all orbital fractures (Table 2).

\section{Treatment modalities}

Surgical treatment was performed on the majority of the patients (67\%) (Table 3). The frequency of surgical treatment increased with age, without statistical significance. The treatment modalities

\section{Table 2. Locations of 164 orbital fractures}

\begin{tabular}{|lc|}
\hline Fracture location & Number of fractures (\%) \\
\hline Medial wall & $78(48)$ \\
Inferior wall & $61(37)$ \\
Inferomedial wall & $25(15)$ \\
Total & $164(100)$ \\
\hline
\end{tabular}

\section{Table 3. Treatment in each age group}

\begin{tabular}{|lcccrrrr|}
\hline \multirow{2}{*}{ Treatment } & \multicolumn{7}{c|}{ Age group (yr) } \\
\cline { 2 - 7 } & $\mathbf{0 - 3}$ & $\mathbf{4 - 6}$ & $\mathbf{7 - 9}$ & $\mathbf{1 0 - 1 2}$ & $\mathbf{1 3 - 1 5}$ & $\mathbf{1 6 - 1 8}$ & Total \\
\hline Surgical & $2(7)$ & $33(70)$ & $42(61)$ & $54(61)$ & $201(75)$ & $167(69)$ & $499(67)$ \\
Nonsurgical & $25(93)$ & $14(30)$ & $27(39)$ & $34(39)$ & $68(25)$ & $74(31)$ & $242(33)$ \\
Total & $27(4)$ & $47(6)$ & $69(9)$ & $88(12)$ & $269(36)$ & $241(33)$ & $741(100)$ \\
\hline Values are presented as numbers (\%). & & & & \\
\hline
\end{tabular}




\section{Table 4. Treatment of fractures}

\begin{tabular}{|lc|}
\hline Fracture/treatment & Number of patients \\
\hline Nasal bone fracture & \\
Observation & 189 \\
Closed reduction & 351 \\
Open reduction & 16 \\
Blow-out fracture & \\
Observation & 61 \\
OWR-absorbable & 89 \\
OWR-metal & 8 \\
Transconjunctiva I approach & 65 \\
Subciliary approach & 31 \\
Zygomaticomaxillary & \\
Observation & 4 \\
O/R-absorbable plate & 31 \\
O/R-metal plate & 7 \\
both & 5 \\
GILLIES approach & 12 \\
Transconjunctiva I approach & 8 \\
Subciliary approach & 16 \\
Mandible fracture & \\
Observation & 7 \\
O/R & 10 \\
IMF with IMF screws & 11 \\
IMF with arch bars & 2 \\
\hline O/R, open reduction; OWR, orbital wall reconstruction; IMF, intermaxillary fixation. \\
\hline
\end{tabular}

Table 5. Mean length of hospital stay in each age group

\begin{tabular}{|lc|}
\hline Age group (yr) & Length of hospital stay (day) \\
\hline $0-3$ & $0.3 \pm 1.3$ \\
$4-6$ & $4.4 \pm 8.3$ \\
$7-9$ & $4.2 \pm 5.2$ \\
$10-12$ & $3.6 \pm 3.8$ \\
$13-15$ & $4.0 \pm 4.8$ \\
$16-18$ & $5.6 \pm 8.8$ \\
Total & $4.7 \pm 6.6$ \\
\hline Values are presented as means \pm standard deviation. \\
\hline
\end{tabular}

are shown in Table 4. Approximately 95\% of nasal bone fractures were treated by closed reduction, while $91 \%$ of orbital wall fractures and $62 \%$ of zygomaticomaxillary fractures were treated by reconstruction with absorbable implants. The average length of hospital stay was 7.3 days with a range of 1 to 43 days (Table 5).

\section{Associated injuries and complications}

Patients with facial fractures often had other concomitant injuries. Associated injuries were seen in $21 \%$ of the children with facial fractures. These included facial lacerations (58\%), neurocranial injuries (23\%), tooth injuries (10\%), extremity fractures (10\%), and eye injuries (9\%) (Table 6). The complication rate of pediatric facial fractures was rare ( $2 \%$ of the 741 patients) (Table 7). The complications included diplopia, facial paresthesia, ectropion, infection, and unsatisfactory fracture repair.
Table 6. Associated injuries

\begin{tabular}{|lc|}
\hline Injury & Number of patients \\
\hline Facial laceration & 90 \\
Brain contusion & 28 \\
Tooth injury+other Fractures & 17 \\
Upper and lower extremity Contusion & 16 \\
Eye injury & 15 \\
Rib/sternum/clavicle & 5 \\
Skull fracture & 5 \\
Lung & 5 \\
Liver and spleen & 5 \\
Intracranial hemorrhage & 3 \\
Cervical spine & 3 \\
Pelvis & 2 \\
Scrotal laceration & 1 \\
\hline
\end{tabular}

Table 7. Postoperative complications of facial fractures

\begin{tabular}{|lc|}
\hline Complication & Number of patients \\
\hline Diplopia & 6 \\
Facial paresthesia & 6 \\
Ectropion & 4 \\
Infection & 2 \\
Unsatisfactory fracture repair & 1 \\
Total & 19 \\
\hline
\end{tabular}

\section{DISCUSSION}

There have been several large studies regarding the epidemiology of facial bone fractures in the literature [7-9]. There is, however, a general lack of information on their clinical features, particularly the incidence and characteristics of facial fractures in the Korean pediatric population. In addition, we analyzed not only the patients who visited the outpatient clinic, but also those who visited the Department of Emergency Medicine. This study was performed at a tertiary center covering a city, several towns, and rural areas, thereby providing a sample that reflects the general population as closely as possible.

The mean age of our study population was 13.0 years, which is consistent with the findings of several other studies $[10,11]$. Similar to previous reports, our study showed a higher incidence of fractures in the 13 to 18 age group (68\%) than in the 0 to 6 age group $(10 \%)[2,4,5]$. Patients aged $>13$ years had developed more adult-like lifestyles. This lifestyle change results from increasing participation in socializing or contact sports, a significantly higher incidence of assaults, and a general decrease in supervision [12]. The male predominance in our study is also in agreement with that of several recent studies [13-15]. Compared to females, males are more likely to sustain trauma and violence due to participation in more physical activities than females. Our 
study also found that boys were more likely to sustain fractures due to violence.

In our study, the monthly distribution of facial fractures peaked in the fall (October) and spring and was lower during summer and winter vacations, which is consistent with the results of a previous report [16]. This distribution may be attributed to children's engagement in new school terms in the spring and fall. Facial fracture occurred most frequently between $1 \mathrm{PM}$ and $6 \mathrm{PM}$. This may be a consequence of the high incidence of violence while commuting home from school.

In our study population, interpersonal violence was the most common type of injury (38\%), followed by falling (31\%), sports (17\%), motor vehicle accidents (8\%) and bicycle accidents (4\%). This differed from the results of previous studies, which reported that road accidents $[1,7,17]$, bicycle accidents [15], and falling [18] were the primary causes. Violence was the most common cause of injury in the 13 to 18 age group, and violencerelated fractures were more common in males, which suggests that the frequency of pediatric fractures is related to the degree of exposure to violence. The nasal bone was the most commonly fractured. As in adults, the reasons for this may be its prominent central position and the delicacy of the nasal bone. Nonetheless, nasal bone fractures are easily missed in children for the following reasons: 1) physical examination is difficult to perform, 2) crepitation of resilient bones may be missing, and 3) edema can conceal the deviation of the nasal dorsum [19]. But some previous studies have indicated that mandibular fractures are the most common facial bone fracture in children $[1,15]$.

Methods of surgical treatment of pediatric facial fractures are quite diverse. Ferreira et al. [5] reported that $78 \%$ of pediatric patients with facial fractures undergo operative fixation, while Imahara et al. [19] reported an operation frequency of only $25.1 \%$. In our study, most patients $(70 \%)$ aged $>3$ years underwent surgery, and absorbable materials were also employed in a large number of patients. The absorbable materials avoided a secondary operation for material removal and prevented failures of bony growth due to the presence of metal materials. Older children required surgery more frequently than younger children, which is in accord with the results of previous studies $[5,19]$. These results may be explained as follows. First, increased mineralization, higher-velocity trauma, decreased fatty padding, and more pneumatized sinuses in older children make displaced bicortical injuries more common. Second, younger children are more frequently indicated for nonsurgical treatment due to their high healing and remodeling capacity or because surgical treatment on the immature skeleton can impair future growth. Third, the growing craniofacial skeleton possesses an inherent plasticity that may not require operative intervention [4].
The characteristics and manifestations of pediatric facial fractures differ among countries, and such differences are influenced by social, educational, and cultural factors. Oh et al. [16] reported, in a study of 201 Korean patients over a period of 10 years, that the incidence of pediatric facial fractures was higher in males (5.5:1), that the most common cause was violence, that the incidence reached the peak in March and November, and that nasal bone fracture was the most common type. These results are consistent with ours. In Japan, Iida and Matsuya [15] demonstrated that facial fractures were more common in males and that violence was more common cause in patients older than 13 years, while in all age groups taken together, bicycle accidents were the most common cause, which may have been associated with Japan's bicycle-based lifestyle. They also mentioned that the prevalence of bicycle accidents significantly decreased probably due to the recent popularity of TV and computer games, and thus resulting in children spending more time indoors than before. In contrast, Ferreira et al. [5] found in a study with 1,251 fractures in Portugal, that pediatric facial fractures were more frequent in males and in the summer, that traffic accidents were a main cause, and that mandible fractures were the most common type. Munante-Cardenas et al. [20] reported, in a study of pediatric patients with facial fractures, that facial fractures more frequently occurred in boys, that bicycle accidents were the most common cause of the fractures, and that mandible fractures were the most common among all types of facial fractures.

The frequency of complications was low in our patients. Postoperative infections, malunion, and nonunion are rare in children because of their greater osteogenic potential, faster healing rate, and less frequent requirement of open reduction and rigid internal fixation. However, the follow-up period of less than 1 year is thought to be too short to draw any conclusions about the effects of trauma and internal fixation on the growing skeleton.

In conclusion, we conducted a retrospective study of facial bone fractures in children and adolescents during the past 5 years. We found that pediatric facial fractures occurred most frequently in males and during the middle school years. In addition, violence was the most common cause of pediatric facial fractures. Since school violence is a serious social problem, we should develop measures to prevent its occurrence. This study provides an overview of facial bone fractures in pediatric age groups, which helps illustrate the trends and characteristics of the fractures. Insight into the epidemiologic data regarding pediatric facial fractures and concomitant injuries is integral to evaluating the quality of pediatric patient care, developing optimal treatment modalities, and making decisions on appropriate resource and manpower allocations. 


\section{REFERENCES}

1. Haug RH, Foss J. Maxillofacial injuries in the pediatric patient. Oral Surg Oral Med Oral Pathol Oral Radiol Endod 2000;90:126-34.

2. Grunwaldt L, Smith DM, Zuckerbraun NS, et al. Pediatric facial fractures: demographics, injury patterns, and associated injuries in 772 consecutive patients. Plast Reconstr Surg 2011;128:1263-71.

3. Murphy RXJr, Birmingham KL, Okunski WJ, et al. Influence of restraining devices on patterns of pediatric facial trauma in motor vehicle collisions. Plast Reconstr Surg 2001;107: 34-7.

4. Vyas RM, Dickinson BP, Wasson KL, et al. Pediatric facial fractures: current national incidence, distribution, and health care resource use. J Craniofac Surg 2008; 19:339-49.

5. Ferreira PC, Amarante JM, Silva PN, et al. Retrospective study of 1251 maxillofacial fractures in children and adolescents. Plast Reconstr Surg 2005;115:1500-8.

6. Losee JE, Afifi A, Jiang S, et al. Pediatric orbital fractures: classification, management, and early follow-up. Plast Reconstr Surg 2008;122:886-97.

7. Pappachan B, Alexander M. Correlating facial fractures and cranial injuries. J Oral Maxillofac Surg 2006;64:1023-9.

8. Alvi A, Doherty T, Lewen G. Facial fractures and concomitant injuries in trauma patients. Laryngoscope 2003;113: 102-6.

9. Kieser J, Stephenson S, Liston PN, et al. Serious facial fractures in New Zealand from 1979 to 1998. Int J Oral Maxillofac Surg 2002;31:206-9.

10. Anderson PJ. Fractures of the facial skeleton in children. In- jury 1995;26:47-50

11. Holland AJ, Broome C, Steinberg A, et al. Facial fractures in children. Pediatr Emerg Care 2001;17:157-60.

12. Shaikh ZS, Worrall SF. Epidemiology of facial trauma in a sample of patients aged 1-18 years. Injury 2002;33:669-71.

13. Gassner R, Tuli T, Hachl O, et al. Craniomaxillofacial trauma in children: a review of 3,385 cases with 6,060 injuries in 10 years. J Oral Maxillofac Surg 2004;62:399-407.

14. Sherick DG, Buchman SR, Patel PP. Pediatric facial fractures: a demographic analysis outside an urban environment. Ann Plast Surg 1997;38:578-84.

15. Iida S, Matsuya T. Paediatric maxillofacial fractures: their aetiological characters and fracture patterns. J Craniomaxillofac Surg 2002;30:237-41.

16. Oh M, Kim YS, Youn HH, et al. Clinical analysis of pediatric facial bone fracture; 10-years experiences in 201 cases. J Korean Soc Plast Reconstr Surg 2005;32:55-9.

17. Arbogast KB, Durbin DR, Kallan MJ, et al. The role of restraint and seat position in pediatric facial fractures.J Trauma 2002;52:693-8.

18. Mericli AF, DeCesare GE, Zuckerbraun NS, et al. Pediatric craniofacial fractures due to violence: comparing violent and nonviolent mechanisms of injury. J Craniofac Surg 2011; 22:1342-7.

19. Imahara SD, Hopper RA, Wang J, et al. Patterns and outcomes of pediatric facial fractures in the United States: a survey of the National Trauma Data Bank. J Am Coll Surg 2008;207:710-6.

20. Munante-Cardenas JL, Olate S, Asprino L, et al. Pattern and treatment of facial trauma in pediatric and adolescent patients. J Craniofac Surg 2011;22:1251-5. 